\title{
Mitochondrial heteroplasmy in vertebrates using ChIP-sequencing data
}

\author{
Thomas Rensch', Diego Villar², Julie Horvath ${ }^{3,4}$, Duncan T. Odom ${ }^{2,5}$ and Paul Flicek ${ }^{1,5^{*}}$
}

\begin{abstract}
Background: Mitochondrial heteroplasmy, the presence of more than one mitochondrial DNA (mtDNA) variant in a cell or individual, is not as uncommon as previously thought. It is mostly due to the high mutation rate of the mtDNA and limited repair mechanisms present in the mitochondrion. Motivated by mitochondrial diseases, much focus has been placed into studying this phenomenon in human samples and in medical contexts. To place these results in an evolutionary context and to explore general principles of heteroplasmy, we describe an integrated cross-species evaluation of heteroplasmy in mammals that exploits previously reported NGS data. Focusing on ChIP-seq experiments, we developed a novel approach to detect heteroplasmy from the concomitant mitochondrial DNA fraction sequenced in these experiments.

Results: We first demonstrate that the sequencing coverage of mtDNA in ChIP-seq experiments is sufficient for heteroplasmy detection. We then describe a novel detection method for accurate detection of heteroplasmies, which also accounts for the error rate of NGS technology. Applying this method to 79 individuals from 16 species resulted in 107 heteroplasmic positions present in a total of 45 individuals. Further analysis revealed that the majority of detected heteroplasmies occur in intergenic regions.

Conclusion: In addition to documenting the prevalence of mtDNA in ChIP-seq data, the results of our mitochondrial heteroplasmy detection method suggest that mitochondrial heteroplasmies identified across vertebrates share similar characteristics as found for human heteroplasmies. Although largely consistent with previous studies in individual vertebrates, our integrated cross-species analysis provides valuable insights into the evolutionary dynamics of mitochondrial heteroplasmy.
\end{abstract}

Keywords: Heteroplasmy, Chromatin immunoprecipitation sequencing (ChIP-seq), mitochondrial DNA (mtDNA), Mitochondrion, Vertebrates

\section{Background}

Mitochondrial DNA (mtDNA) forms a circular molecule, which is located in the mitochondrial matrix [1]. In mammals, mtDNA is $\sim 16.5 \mathrm{~kb}$ long and contains 37 genes [2]. For the most part, mtDNA either codes for proteins or for ribosomal RNAs and transfer RNAs, except for a $1 \mathrm{~kb}$ stretch known as the control region, which contains one origin of replication and both origins of transcription [3,4]. Several identical mtDNA copies (between 2 and 10 in humans) are present in each

\footnotetext{
* Correspondence: flicek@ebi.ac.uk

${ }^{1}$ European Molecular Biology Laboratory, European Bioinformatics Institute,

Wellcome Genome Campus, Hinxton, Cambridge CB10 1SD, UK

${ }^{5}$ Wellcome Trust Sanger Institute, Wellcome Genome Campus, Hinxton,

Cambridge CB10 1SA, UK

Full list of author information is available at the end of the article
}

individual mitochondrion, which means a single cell can contain hundreds to thousands of copies of mtDNA $[2,4]$. The mtDNA was the first part of the human genome to be sequenced and to this day is one of the most studied segments of DNA in humans and in many other species [2, 4]. In addition to its high copy number, the mutation rate of mtDNA is significantly higher than that of nuclear DNA [3]. These properties make it common for an individual to have more than one mtDNA variant: this phenomenon is known as heteroplasmy [5] and has been observed and studied in many species and contexts.

In humans, hundreds of diseases are linked to point mutations in the mitochondrial genome [6], suggesting that a fraction of human mitochondrial mutations may be pathogenic. Many of these mutations exist in a 
heteroplasmic state and the extent of the disease symptoms vary according to the proportion of the deleterious allele [7]. Such diseases include many metabolic diseases, age-related neurodegenerative diseases such as Alzheimer's and Parkinson's, as well as several types of cancer [6, 8-11]. Research in fields such as population genetics and forensics has also focused on heteroplasmy as a way to investigate aspects of inheritance [12]. Although heteroplasmic positions have also been observed in other mammalian species [13-16], studies of the phenomenon in other taxa have mostly been conducted for genetic barcoding or to investigate molecular evolution and generally focused on small controlled datasets [17-21]. Cross-species comparisons have been reported in a limited number of closely related species, such as different types of bees [22-24].

Heteroplasmy was first reported in 1983 [25] and has been detected with a variety of methods including Sanger capillary sequencing [26] and pyrosequencing [27]. However, these sequencing methods are expensive and slow, which limited the number of studied samples. More recently, next-generation sequencing (NGS) has been used to study mitochondrial heteroplasmy with high-throughput data and several computational approaches for heteroplasmy detection have been developed [28-33]. The main challenge in using NGS data to detect heteroplasmies is sequencing errors, which tend to be location-specific and thus can be confused with heteroplasmies. To avoid such biases, criteria for NGSbased heteroplasmy detection were developed using PhiX genome simulations and establishing different quality thresholds to identify heteroplasmic positions [28]. Since the heteroplasmy detection power increases with coverage, recent studies employing high coverage sequencing $(>1000 \times)$ have adapted these criteria [29] (e.g. more lenient thresholds) as well as developed advanced probabilistic models to detect micro-heteroplasmies (i.e. positions with a minor allele ratio below 2-5 \%) [30, 34]. In this study, we focus on detecting a higher level of heteroplasmy $(>15 \%)$ using a modified version of the established criteria [28].

Previous heteroplasmy studies used targeted mtDNA sequencing. In most cases mtDNA was extracted from whole blood or buccal tissue, although recently a few studies have investigated a range of tissues [35, 36]. More recently, mitochondrial heteroplasmy has been assayed using data from many whole-genome sequencing studies including from the 1000 Genomes Project [37] and in other NGS datasets, such as exome- and RNA-sequencing (RNA-seq) $[38,39]$. It is currently unknown whether genomic enrichment assays such as Chromatin immunoprecipitation followed by high-throughput sequencing (ChIP-seq) have suitable characteristics for mitochondrial heteroplasmy detection including relatively uniform coverage and appropriate sequencing depth, although some assays such as ATAC-sequencing (ATAC-seq) are known to include a high fraction of mtDNA reads.

As the cost of sequencing continues to drop, the quantity of datasets being generated and stored is rapidly increasing. Among the benefits of public availability of sequencing experiments is their use to efficiently answer research questions not explored at the time of data generation. Here, we exploit a combination of previously generated and novel datasets resulting from ChIP-seq experiments to perform heteroplasmy detection across a range of vertebrate species. Although mtDNA is of the order of $0.1 \%$ of all DNA in a cell [40], the high copy number of the circular mitochondrial genome generally leads to it being sequenced many times in ChIP-seq experiments, resulting in a significant proportion of ChIP-seq reads covering the mtDNA [41-43].

We first confirm the prevalence of mtDNA in published ChIP-seq data and show that mtDNA coverage is suitable for heteroplasmy detection. We then apply a novel heteroplasmy detection method to a collection of both novel (see "Methods") and previously published ChIP-seq datasets comprising a total of 79 individuals from 16 species. Our findings provide several insights into the dynamics of mtDNA heteroplasmy over a large portion of the mammalian phylogeny.

\section{Results}

\section{Large mammalian dataset}

We gathered ChIP-seq data from five previously published studies [44-48] and performed new transcription factor (TF) and histone modification ChIP-seq experiments (see "Methods") on a selection of samples that were used in the aforementioned papers. We selected published ChIP-seq datasets (signal and input files) from large cross-species comparison studies to mitigate batch effects. The combined data cover a wide range of species spanning the mammalian clade including primates, rodents, and domesticated animals such as dogs, cats, and cattle, as well as chicken as an out-group vertebrate species. Most of these samples come from liver tissue, but some consist of lymphoblastoid cell lines. After analysis (see below and "Methods"), we identified a core set of 16 species for comparison - Homo sapiens (human), Macaca mulatta (macaque), Chlorocebus aethiops sabaeus (vervet), Callithrix jacchus (marmoset), Otolemur garnettii (bushbaby), Mus musculus domesticus (mouse), Rattus norvegicus (rat), Heterocephalus glaber (naked molerat), Oryctolagus cuniculus (rabbit), Bos taurus (cattle), Delphinus delphis (dolphin), Sus scrofa (pig), Canis familiaris (dog), Mustela putorius furo (ferret), Sarcophilus harrisii (Tasmanian devil), and Gallus gallus (chicken). 


\section{ChIP-sequencing data for heteroplasmy detection}

A ChIP-seq study generally consists of two experiments that each result in short read sequencing data files [49]. The first is commonly known as the signal file and contains reads resulting from the ChIP experiment, which when mapped to the target genome produces read clusters (peaks) identifying genomic locations where the proteins targeted by the ChIP antibody were bound. The second data file is a control experiment consisting of a similar process, but without the immunoprecipitation step, and is used to control for biased genome-wide read coverage arising from preferential sonication of open chromatin [50]. Thus, the control data generally contain reads that map to the entire genome, with few expected enriched regions. Due to the high copy number of the mtDNA, reads within the mitochondrial genome are sequenced many times in both signal and control ChIPseq experiments. We observed significant read coverage in both data files, even though binding peaks within mtDNA were not detected (see "Methods").

We used ChIP-seq data originally generated to map various histone modifications and TFs such as CEBPA and FOXA1, as well as the input/control data for each experiment (Fig. 1). We merged all experiments corresponding to the same individual, which provides better total coverage and acted as technical replicates (i.e. sequencing of the same biological tissue) for the mtDNA detection experiment. When combined in this way, coverage for each individual was relatively high and homogenous for almost all species, with average mitochondrial coverage above $50 \times$ and coverage ratio above $70 \%$ (Fig. 2). As previously reported [28], such coverage levels are adequate to detect high-level heteroplasmies with high specificity. Detailed coverage data for each individual are available in the supplementary materials (Additional file 1: Figure S1). For six species in the original published studies forming our collected dataset, but not included in the 16 core species (Monodelphis domestica, Cavia porcellus, Tupia belangeri, Balaenoptera borealis, Mesoplodon bidens, and Lagenorhynchus albirostris), we observed very low rates of uniquely mapping reads on mtDNA. C. porcellus (guinea pig) and $T$. belangeri (tree shrew) have highly fragmented genome assemblies that may hinder accurate mtDNA read mapping, and $M$. domestica (opossum) is known to have a significantly increased number of NUMTS (nuclear mitochondrial DNA sequences), which may also have affected the number of uniquely mtDNA mapping reads [51]. Samples from Balaenoptera borealis (sei whale), Mesoplodon bidens (Sowerby's beaked whale), and Lagenorhynchus albirostris (white-beaked dolphin) were all mapped to the closely related Tursiops truncatus (common bottlenose dolphin) species' genome, a process that also yielded few uniquely mapping reads to the mtDNA except for Delphinus delphis (short-beaked common dolphin), see Fig. 2. Since coverage in these species was insufficient for heteroplasmy detection, we excluded them from further analysis. Finally, we also

\section{A Data}

Novel \& publicly available ChIP-seq dataset comprising 79 individuals and 16 species
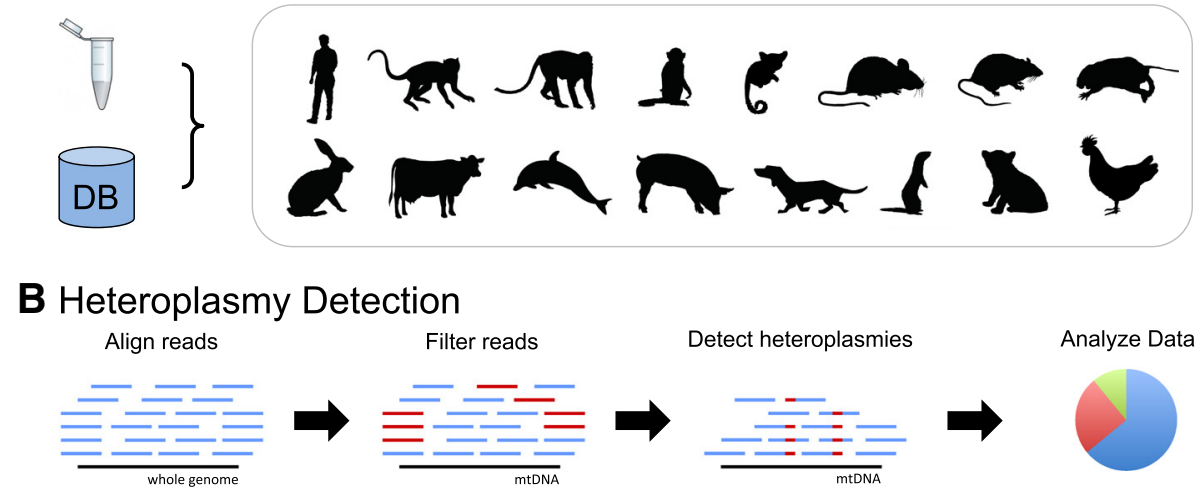

\section{Results}

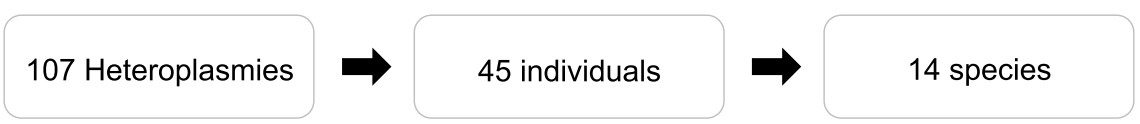

Fig. 1 Heteroplasmy detection workflow. The raw read files obtained from the ChIP-seq experiments were first aligned to the respective reference genomes. The aligned reads were then pre-processed, filtering out duplicate reads and extracting reads mapping with a high quality score to the mtDNA. The heteroplasmy detection algorithm was then used across the samples. Finally, we analyzed the genomic properties of heteroplasmic positions across vertebrates 


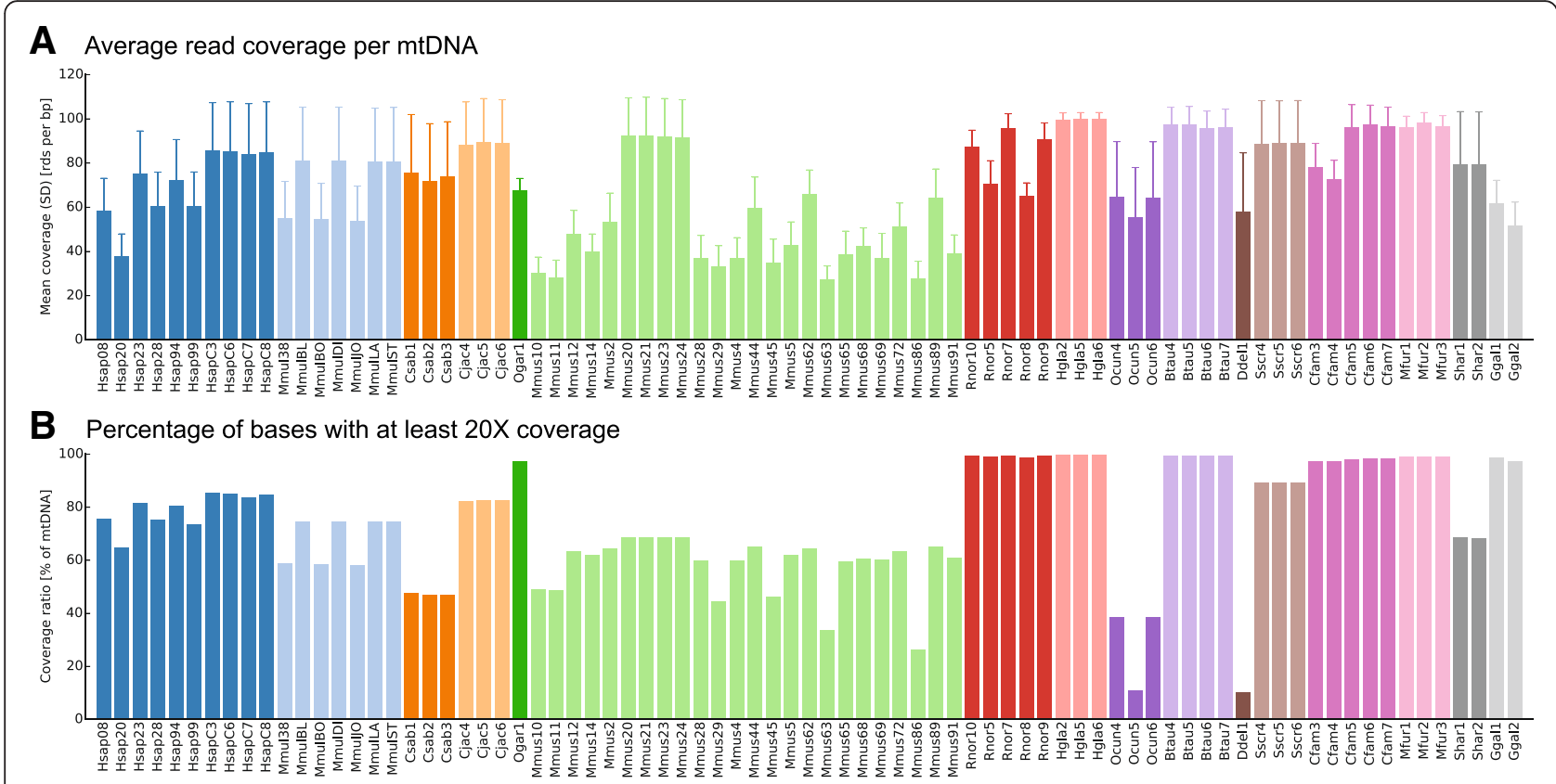

Fig. 2 mtDNA read coverage per individual. a The mean read coverage per mtDNA base pair for each analyzed individual, colored per species (the error bars represent the standard deviation). $\mathbf{b}$ The fraction of mtDNA base pairs covered by at least 20 reads (our heteroplasmy detection cutoff) also colored by species

discarded a Mus musculus (mouse) individual for which the coverage ratio fell below $10 \%$.

\section{Heteroplasmy detection algorithm}

We adapted a previously published heteroplasmy detection methodology [28] for the specific characteristics of ChIP-seq data (see below and "Methods"). Briefly, this method is based on a set of criteria to be checked for each mtDNA base pair. In addition to quality thresholds, the algorithm requires a minimum number of reads to be present on each strand. Verification of heteroplasmies on both strands avoids location-specific errors that may arise from sequencing errors, since it is uncommon for these to occur at the same location on each strand [28]. Distinguishing characteristics of our method include aligning with BWA [52] (instead of assembling the reads) and a parameter set optimized for ChIP-seq data. This parameter set is generally more stringent than in previous reports $[28,29]$ and includes a higher base quality threshold (base quality $>23$ ) and an added minimum coverage threshold (20 reads). We also increased the minimum heteroplasmy level to $15 \%$ (minor allele frequency). Although these changes to the algorithm result in lower expected sensitivity, we do so to optimize specificity from the generally less homogenous sequencing coverage in ChIP-seq samples, compared to that observed in targeted mtDNA resequencing.

Nucleotide repeats present in low complexity regions strongly hinder sequencing quality over those locations.
While previous studies have excluded these positions from their analysis, these regions are also more likely to harbor heteroplasmies due to error-prone polymerase activity and limited DNA repair in the mitochondrion. The human mitochondrial annotation database MITOMAP [53] lists several positions as heteroplasmic within these regions, of which we find nine (see "Methods") with our detection method. Since the detection parameters are very stringent, we decided to keep the repetitive regions in our analysis.

In addition to liver samples, we also applied our detection algorithm to ChIP-seq data from several primate species' lymphoblastoid cell lines (LCLs) [46]. We observed that $33 \%$ of them expressed more than 25 heteroplasmic positions, which we assume might be due to genomic instability in the immortalized cell lines that could have arisen from a high passage number of the cells [54]. Our results from two Felis catus (cat) samples also exhibited a surprisingly high number of heteroplasmies in both individuals (Additional file 2: Table S1). Furthermore, almost all of the positions detected in one cat individual were present in the other, which may be due to low genetic diversity in the source population or the two individuals being siblings. Other possibilities are that some contamination occurred in the process or that cat may have a specific pattern of NUMTS that impedes analysis. For these reasons, we do not include the primate LCLs or the cat data in the core set of species or the remaining comparative analysis in the paper. 
After the filtering above, our final dataset included comparative heteroplasmy results from liver samples of 79 individuals across 16 species. For these, we found 107 positions in 45 individuals across 14 species (Fig. 3; Additional file 2: Table S2). A total of $57 \%$ of the individuals express heteroplasmy. Our estimate is higher than initial NGS-based reports of human heteroplasmy [28], but consistent with recent reports on highcoverage datasets [34, 35], which also showed that liver tissue has one of the highest relative number of heteroplasmies compared to other human tissues [35]. In fact, we find heteroplasmic positions in every species except Heterocephalus glaber (naked mole-rat) and Delphinus delphis (short-beaked common dolphin).

\section{Heteroplasmic positions present in multiple individuals}

Several positions occur in more than one individual of the same species (Fig. 4; Additional file 2: Table S2), a phenomenon that has previously been observed in humans and attributed in part to differential mutation rates across the mtDNA sequence [28]. Since heteroplasmies in humans are mostly located at positions with high relative mutation rate $[28,34]$ and mutation rate patterns are shared across individuals, such positions are more likely to exist in a heteroplasmic state in more than one individual. Similar differential mutation rate patterns are likely in other species and thus the shared positions we observe may also have high mutation rates. Likewise, we asked whether this phenomenon could be linked to sequence conservation, but the heteroplasmic positions occurring in more than one individual do not show evidence of conservation bias (Fig. 4). That some individuals may be closely related via their breeding history is another possible explanation.

\section{Read coverage of the heteroplasmic positions}

The average number of reads supporting each of our observed heteroplasmic position is 60 (SD 25), which is significantly higher than our 20 read threshold (Additional file 1: Figure S2). Indeed, there are no observed positions with read coverage of exactly 20 reads and only three positions with coverage of 21 reads. Based on the observed coverage distribution, our detection parameters including the coverage threshold appear to be conservative.

For each individual, we compared the average coverage across the mtDNA to the number of observed heteroplasmic positions. We found a minor correlation across the entire distribution (Pearson's $r=0.17$ ) (Fig. 5a), but for individuals with high average coverage ( $>40$ reads per mtDNA position), there is essentially no correlation between coverage and number of observed heteroplasmies (Pearson's $r=0.05$ ). This result further suggests that our chosen coverage threshold is sufficient for robust detection of high-level heteroplasmies. As expected, the heteroplasmy level distribution is highest at $15 \%$ minor allele frequency corresponding to the threshold level (Fig. 5c). Previous studies also report that the majority of heteroplasmic positions occur at the lowest minor allele level [28, 34].

\section{Heteroplasmy mutation spectrum analysis}

The transition-transversion rate is strongly biased in the mitochondrion [55]. We observe a transition-transversion ratio of $\mathrm{Ts} / \mathrm{Tv}=3.86$ in our results across all species, which is similar to the ratio found in the MITOMAP database ( $\mathrm{Ts} / \mathrm{Tv}=2.95$, Fisher's exact test, $p=0.31)$. In fact, the full mutational spectrum we observe in our multi-species dataset (Fig. 5b) is similar to that observed in the

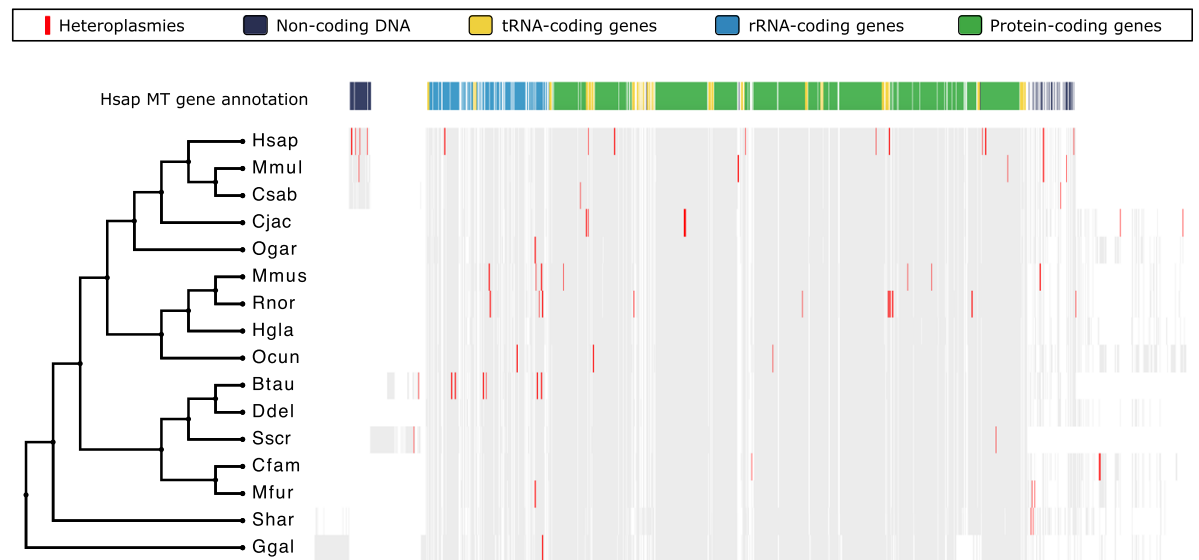

Fig. 3 Heteroplasmies in 16 species. Detected heteroplasmy in H. sapiens (human), M. mulatta (macaque), C. sabaeus (vervet), C. jacchus (marmoset), O. garnettii (bushbaby), M. musculus (mouse), R. norvegicus (rat), H. glaber (naked mole-rat), O. cuniculus (rabbit), B. taurus (cattle), D. delphis (dolphin), S. scrofa (pig), C. familiaris (dog), M. putorius furo (ferret), S. harrisii (Tasmanian devil), and G. gallus (chicken) displayed in red on the mtDNA multiple alignment with the associated evolutionary tree. The human gene annotation displayed at the top of the figure shows RNA and protein-coding genes as well as non-coding regions 


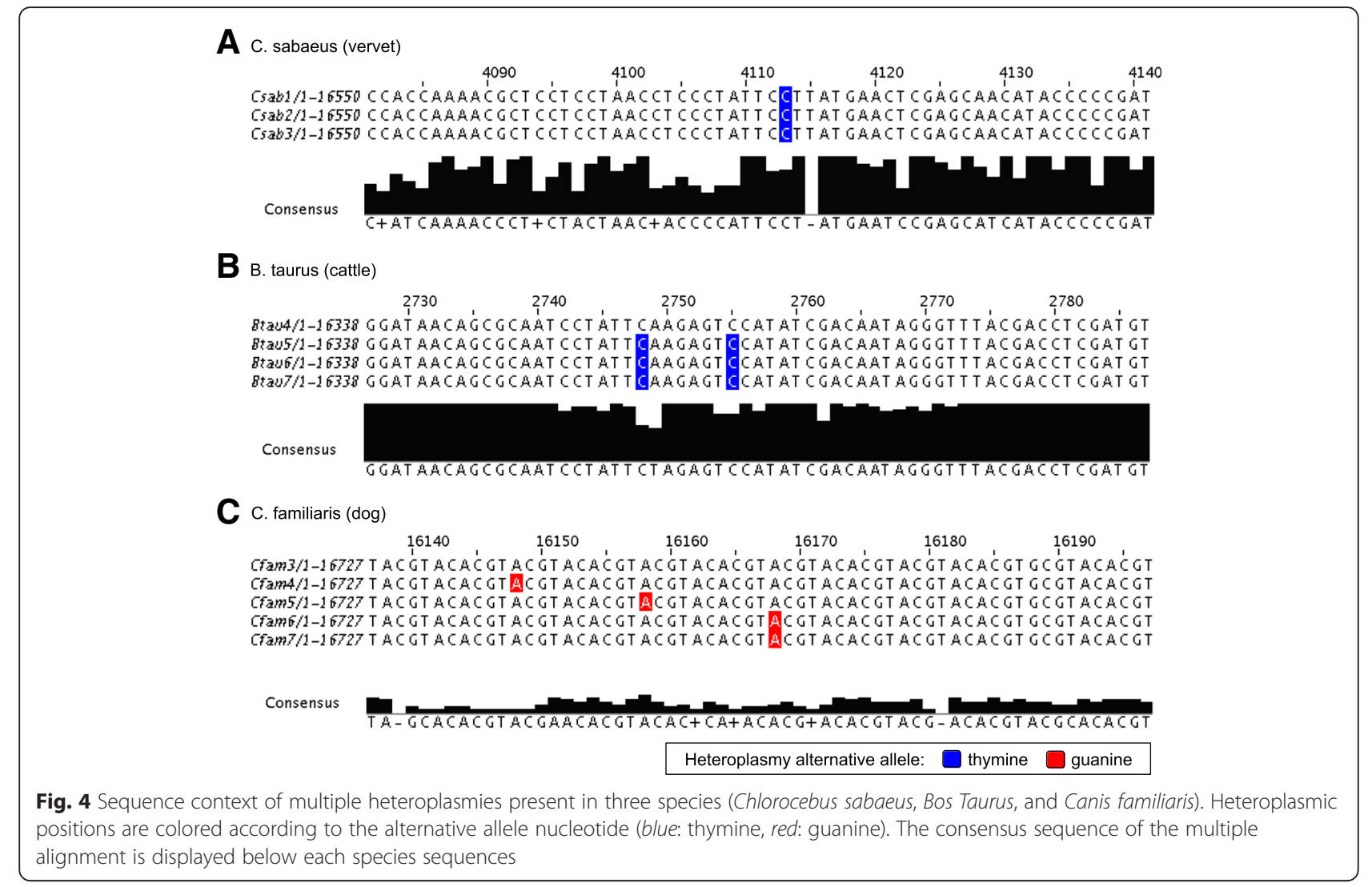

MITOMAP database $\left(X^{2}\right.$ test, $\left.p=0.07\right)$. Additionally, the fact that the most common Illumina sequencing errors (AC and GT transversions [56]) are rare in our observed multi-species mutation spectrum strongly suggests that we have few false positives due to sequencing errors in our set of heteroplasmic positions.

\section{Genomic location of the detected heteroplasmies}

As shown in Figs. 3 and 5d, out of the 107 heteroplasmic positions found, 44 are in intergenic regions with most of these in the hyper-variable D-loop and only five in other mitochondrial intergenic regions. Of the remaining positions, 39 are located in non-protein-coding genes (28 in ribosomal RNA (rRNA) genes and 11 in tRNA genes) and 24 positions are located in protein-coding genes. Of the protein-coding gene changes, 13 are synonymous variations meaning they do not affect the amino acid used in the translated protein, while there are 11 non-synonymous variants in six species. However, most of the observed amino acid changes (7 out of 11) are between residues with similar biochemical properties. In four cases, the observed changes from isoleucine to threonine and from valine to alanine are modifications between hydrophobic and hydrophilic amino acids.

The relative paucity we observed for heteroplasmies in non-coding regions of the mitochondrial genome may be partly due to negative selection acting on heteroplasmy positions within mitochondrial genes [33].

\section{Heteroplasmic positions associated with disease}

For humans, about $5 \%$ of mtDNA positions are associated with disease [53]. Using the MITOMAP annotations, five positions (15\%) among the human heteroplasmies we find are disease-associated. This is more than the proportion of positions associated with disease in MITOMAP, but comparable to the previously observed proportion in humans from a set of five Eurasian populations (11.8 \%, Fisher's exact test, $p=0.99$ ) [28]. We then considered whether a similar proportion of positions in other species could be considered deleterious. Since there are no MITOMAPtype databases for the other species listing disease associations, we assigned heteroplasmic positions in other species to their orthologous human positions (see "Methods"). For the 43 positions in other species that could be confidently assigned an orthologous position on the human mitochondria, two $(4.7 \%)$ were associated with disease (13,882 in Rnor5 - Rattus norvegicus and 1068 in Btau4 Bos taurus) based on human MITOMAP annotations. These correspond to a synonymous protein-coding gene mutation in Rattus norvegicus and an rRNA mutation in Bos Taurus. Although we do not assume that disease 


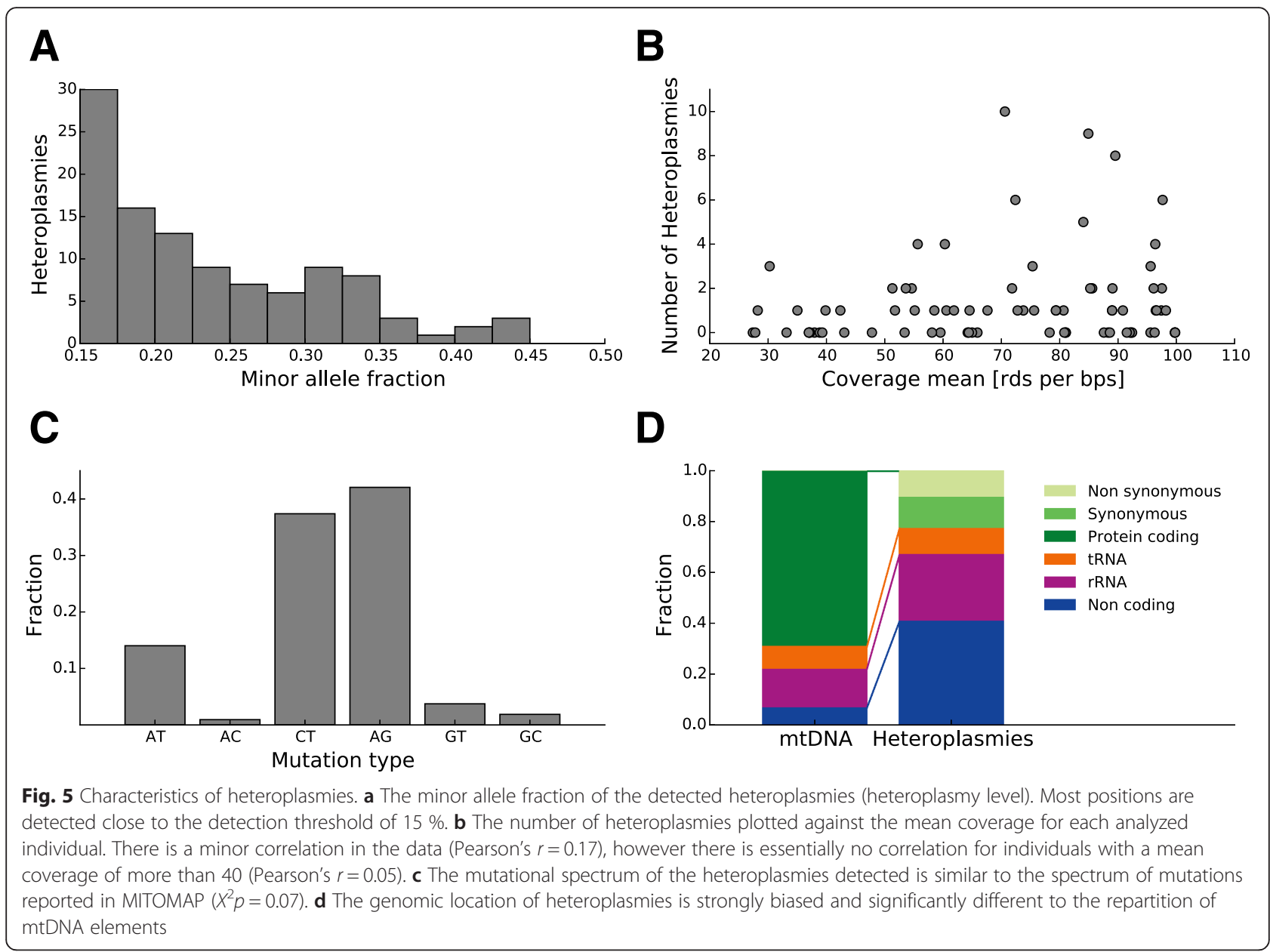

associations observed in humans will be maintained at orthologous positions in other species, the observed rate is indistinguishable from the baseline disease associated rate in the MITOMAP database (Fisher's exact test, $p=0.99$ ). Therefore, these results suggest that the distribution of functionally relevant mitochondrial mutations is similar across the species we studied. This observation may reflect a comparable mitochondrial disease burden across mammals.

\section{Sanger sequencing and pyrosequencing validation of heteroplasmies}

To validate the mitochondrial heteroplasmies identified in ChIP-seq datasets with an independent method, we selected 34 heteroplasmic positions randomly from the total 107 we detected (see Additional file 2: Table S3). This validation set includes positions with a range of minor allele frequencies and comprises 12 species. For these, we performed Sanger sequencing on mtDNA amplicons to confirm the presence of two sequence variants at each heteroplasmic position (see
"Methods"). We validated heteroplasmies with high confidence in 14 positions. A further seven positions showed some evidence for heteroplasmy, while we did not detect any heteroplasmy in the remaining 14 positions (41\%). Because of the relatively low sensitivity of Sanger sequencing for detection of single-nucleotide variants [57], we performed further validations using pyrosequencing as an alternative method. We selected five of the previously tested positions we could not validate by Sanger sequencing and an additional 13 positions (Additional file 2: Tables S3 and S5). Pyrosequencing data supported heteroplasmies predicted from ChIP-seq data for all of the positions previously tested by Sanger sequencing and 11 out of the 13 (85\%) newly tested positions. For three of the newly validated positions, the identities of minor and major allele were reversed in pyrosequencing compared to the ChIP-seq based detection, which may be due to indels or other discrepancies between ChIP-seq libraries and mtDNA samples used for pyrosequencing. In sum, these results indicate that our stringent criteria 
for heteroplasmy detection in ChIP-seq experiments largely identify true mitochondrial heteroplasmies that can be independently validated by Sanger sequencing or pyrosequencing of mtDNA samples.

Along with the validation of heteroplasmic sites identified in ChIP-seq data, we also performed pyrosequencing of the same positions in different individuals of the corresponding species for which our NGS method did not detect heteroplasmies. Supporting the stringency of our computational approach, in a number of cases pyrosequencing data also detected low levels of heteroplasmy in individuals where ChIP-seq based identification was negative (see Additional file 2: Tables S3 and S5).

\section{Discussion}

\section{Detected heteroplasmic positions}

We find an average number of 2.4 heteroplasmic positions per individual with heteroplasmy. Although this is higher than previously found in humans [28, 29], two recent studies show that liver tissue has an increased amount of heteroplasmies compared to other tissues, and most previous studies were performed with whole blood and buccal tissue samples $[35,36]$. Our detection method also extends prior approaches by not discarding heteroplasmic positions within low complexity regions (see "Results"), while these were not considered in previous studies. Moreover, we find most heteroplasmic positions with minor allele ratios close to our $15 \%$ threshold (Fig. 5c). This is consistent with previous studies, which also found most heteroplasmies at their respective thresholds [28, 34], and suggests that additional heteroplasmies exist beyond the threshold that we used. Finally, since we used relatively stringent detection criteria, it is likely that the heteroplasmic positions we identified are in fact a subset of all heteroplasmies present in the samples. We hypothesize that the apparent absence of heteroplasmies in Heterocephalus glaber (naked mole-rat) and Delphinus delphis (short-beaked common dolphin) is due to profiling of only a few individuals in these species (one individual of $D$. delphis and three of $H$. glaber).

The genomic location of heteroplasmies across vertebrates is also consistent with previous findings [28, 34, 36] with the majority of positions occurring in the non-coding control region which is the most polymorphic segment of the mtDNA. In fact, most of the heteroplasmies are located in non-coding regions or (structural) RNA genes in which modifications of single nucleotide bases are expected to only have minor effects [58]. Furthermore, heteroplasmies located in protein-coding genes, genome elements that are highly conserved [59], are almost exclusively either synonymous mutations or result in biochemically similar amino acids.

\section{Validation of heteroplasmies identified in ChIP-seq datasets}

Through a combination of Sanger sequencing and pyrosequencing, we carried out validation experiments for a total of 47 heteroplasmies predicted from ChIP-seq data. While Sanger sequencing alone only validated 20 out of 34 interrogated positions (59\%), a fraction of the undetected positions could still be true heteroplasmies given the low sensitivity of this method. Further validation using pyrosequencing as a more sensitive method further validated heteroplasmies that were negative in Sanger sequencing ( 5 out of 5 tested), as well as a large fraction of newly tested heteroplasmy positions detected at low minor allele frequencies in ChIP-seq data (11 out of $13,85 \%)$. These results strongly suggest that most of the heteroplasmies we identified in ChIP-seq data are true heteroplasmies that can be validated in mtDNA samples. However, given the stringency of our detection method (with a minor allele threshold of $15 \%$ ), it remains likely that we are underestimating the full extent of heteroplasmy positions in each sample. In this regard, mtDNA samples from individuals for which a particular heteroplasmy could not be detected based on our ChIPseq method often showed low levels of heteroplasmy in pyrosequencing.

\section{Number of different mtDNA genomes}

When an individual expresses one heteroplasmic position, it is likely that only two variants of the mtDNA genome exist within its cells. However, when individuals express more than one heteroplasmic position, which is the case for 21 individuals in this study (see Additional file 2: Table S2), we cannot determine the underlying number of mtDNA genome variants. Estimating this number could be possible using the heteroplasmy minor allele fractions, however this would require a precise evaluation of the heteroplasmy level. This might be feasible with high coverage data but is not realistic with the data presented here. Further, none of the detected heteroplasmic positions has more than two alleles. Although our analysis revealed a handful of positions that have one sequencing read containing an additional third base, such potential third alleles never fulfill the criteria to be determined as alternative minor alleles. In sum, our results cannot discriminate the underlying number of mtDNA genomes. Emerging technologies that promise to provide significantly longer reads would help in identifying combinations of mutations on the same mtDNA molecule.

\section{MtDNA coverage in ChIP-seq data}

It is well-known that the mtDNA is sequenced many times due to its high copy number and that significant amounts of mtDNA reads are found in most NGS 
generated datasets including in ChIP-sequencing data for which mtDNA coverage has even been used as a control for background noise [41]. However, since most ChIP-seq studies focus on binding events in the nuclear DNA and use other control methods, reads mapping to mtDNA have been discarded as mitochondrial contamination. Here we demonstrate that mtDNA coverage in ChIP-seq samples is in fact so high that it may be used similarly to targeted mtDNA shotgun sequencing. We first studied the coverage of each ChIP-seq file independently and observed that it was consistently deeper in the control files compared to the TF binding files, which also had higher mtDNA coverage than the histone ChIP-seq files (Additional file 1: Figure S3). Low mtDNA coverage in ChIP-seq for histone marks is expected, since histones are not used to pack mtDNA in the mitochondrion [5]. Although the investigated TFs do not show signs of binding on the mtDNA (see "Methods"), it has been reported that several TFs that are active in the nucleus can also bind the mtDNA [43] and such weak binding events could explain the high coverage found in our experiments. However, due to the even read coverage observed, it seems most likely, at least for the factors investigated here, that the presence of mtDNA in ChIPsequencing data is a technical and not biological phenomenon. Overall, after merging the different files the coverage level and ratio were relatively high and comparable to the data from Li et al. [28], on which we based our detection algorithm. On the other hand, coverage ratio was variable between species, ranging from the entire mtDNA to as little as $10 \%$. This caveat restricts the conclusions we can draw about the genomic location of heteroplasmies within individual species, since some portions of the mtDNA genome are not covered in every individual. However, although we were limited in our ability to conduct some analyses, such as detailed cross-species comparisons of heteroplasmic positions, we focused on extracting information from our dataset as a whole to obtain conclusive results.

\section{ChIP-seq datasets are useful data sources for the study of heteroplasmy}

We have shown that it is possible to make use of ChIPsequencing data that were originally collected for other purposes to explore mitochondrial heteroplasmies across a wide variety of mammalian and vertebrate species, by taking advantage of the high number of sequenced mtDNA reads in these experiments. We hope our general approach will encourage the further study of mtDNA and other biological questions using valuable existing datasets. For instance, making use of data arising from other profiling-sequencing methods such as RNA-seq or ATAC-seq might be very useful, as demonstrated by a recent study of the mitochondrial transcriptome [38]. However, making use of RNA-seq data to explore mitochondrial heteroplasmy would require caution, as some RNA-DNA differences (RDDs) have been reported in the mitochondrial genome [60]. Finally, although we adapted a detection method appropriate for our datasets of relatively low-coverage and variable homogeneity, higher coverage and/or more advanced methods for heteroplasmy detection [30, 34] could potentially be valuable for a wider variety of existing data types. For example, higher coverage datasets will likely enable new methods to explore lower levels of heteroplasmy in ChIP-seq data. Furthermore, having demonstrated that ChIP-seq coverage of the mtDNA can be substantial and allows for the study of heteroplasmy, it is likely that ChIP-seq data can be used to perform other mtDNA studies in fields such as population genetics, forensics, and in some areas of medical research. There is a current trend of creating large genotype-phenotype datasets for ChIP-seq and RNA-seq data and using these datasets to explore phenotype associations to heteroplasmies could prove extremely valuable to further our knowledge of the phenomenon.

\section{Conclusions}

\section{Heteroplasmies show consistent characteristics}

Our study shows that mitochondrial heteroplasmy displays similar characteristics across vertebrate species, including genomic location and mutation spectrum across the vertebrate species tree. As might be expected, our results strongly suggest that previous heteroplasmy findings established in humans are valid for all mammals and possibly all vertebrates. In addition to this, our results also support recent findings that heteroplasmy is more prevalent in liver compared to other tissues $[35,36,61]$. Our results also suggest that any new understanding about heteroplasmies will likely apply across the mammalian clade. It is clear, both in our findings and in previous work, that mutation rates vary significantly between positions, meaning some positions are more likely to exist as heteroplasmies than others. Although there is limited information, on a molecular level, describing the functional impacts these heteroplasmies may have, our results suggest that those functional impacts are likely to be similar across many different mammalian species and that many species may be effective model organisms for understanding the biology of heteroplasmy.

\section{Methods}

\section{ChIP-seq data}

We used a combination of newly generated and previously published ChIP-seq data as described here.

We performed ChIP-seq experiments for CEBPA, H3K4me1, H3K4me3, H3K27ac, and total histone H3 on 
a collection of liver samples from multiple species: CEBPA in nine species (C. jacchus, $C$. familiaris, $C$. porcellus, F. catus, $H$. glaber, M. domestica, M. furo, O. cuniculus, and $T$. belangeri), H3K4me1 in 20 species (B. borealis, B. taurus, C. jacchus, C. familiaris, C. porcellus, C. sabaeus, D. delphis, F. catus, H. glaber, L. albirostris, M. mulatta, M. bidens, M. domestica, M. furo, O. cuniculus, O. garnettii, R. norvegicus, S. harrisii, S. scrofa, and $T$. belangeri), H3K4me3 in three species (C. jacchus, O. garnettii, and O. garnettii), H3K27ac in six species (H. glaber, M. domestica, O. garnettii, S. harrisii, S. scrofa, and T. belangeri), and total histone H3 in eight species (D. delphis, H. glaber, M. mulatta, M. domestica, $O$. garnettii, S. harrisii, S. scrofa, and T. belangeri). Additional details are listed in Additional file 2: Table S4.

Wherever possible, livers from young adult males were used. Tissues from eight species were excess from routine euthanasia procedures including from individuals sacrificed during maintenance of research colonies. Five species were purchased commercially from slaughterhouses, for example. Specialty conservation programs including zoos, the Duke Lemur Center, or Cetacean Strandings surveillance often collect tissues for research purposes, and we obtained seven species' tissues from these efforts. With the exception of the Lagenorhynchus albirostris sample, cetacean tissues were from live stranded individuals that died on the beach and were in a freshly dead condition at the time of post-mortem.

Our ArrayExpress [62] submission, E-MTAB-3933, has complete sample meta-data, raw sequencing files, and detailed experimental protocols. Briefly, chromatin immunoprecipitation was carried out from $0.1-0.5 \mathrm{~g}$ of liver tissue, using antibodies against H3K4me3 (millipore 05-1339), H3K27ac (abcam ab4729), H3K4me1 (abcam ab8895), total histone H3 (abcam ab1791), or CEBPA (Santa Cruz Biotechnology sc-9314). Histone mark ChIP experiments were performed with automated 96-well protocols in an Agilent Bravo liquid handling robot [63]. A manual version of the protocol was used for CEBPA experiments to allow for higher chromatin input.

The previously published data obtained from [44-48] was obtained from the ArrayExpress database (see "Supporting Data" for accession numbers). We performed the preprocessing and aligning of the reads (see below) so only raw read files (in FASTQ format) were downloaded. Details of the datasets are as follows: HNF4A and CEBPA ChIP-seq data for the liver tissue of five species (H. sapiens, M. musculus, C. familiaris, M. domestica, and G. gallus) [44]; CTCF, SA1, NRSF/REST, and H2AK5ac ChIP-seq data for the liver tissue of six species (H. sapiens, $M$. mulatta, $M$. musculus, $R$. norvegicus, $C$. familiaris, and M. domestica) [45]; CTCF and YY1 ChIP-seq data for LCLs of seven species (H. sapiens, P. troglodytes, G. gorilla, $P$. pygmaeus, M. mulatta, $P$. hamadryas, and $S$. oedipus), and YY1 liver tissue ChIP-seq data for two species (H. sapiens and M. musculus) [46]; CEBPA, FOXA1, ONECUT1, and HNF4A ChIP-seq data for the liver tissue of five species ( $H$. sapiens, M. mulatta, M. musculus, $R$. norvegicus, and C. familiaris) [47]; H3K4me3 and H3K27ac ChIP-seq data for the liver tissue of 21 species (H. sapiens, M. mulatta, C. sabeus, C. jacchus, M. musculus, $R$. norvegicus, C. porcellus, $H$. glaber, O. cuniculus, T. belangeri, B. taurus, D. delphis, L. albirostris, B. borealis, M. bidens, S. scrofa, C. familiaris, F. catus, M. furo, $M$. domesticus, and S. harrisii) [48]; and CEBPA, H3K4me1, H3K27ac, and total Histone H3 ChIP-seq data for the liver tissue of 21 species (M. mulatta, C. sabeus, C. jacchus, $M$. musculus, $R$. norvegicus, $C$. porcellus, $H$. glaber, O. cuniculus, $T$. belangeri, $B$. taurus, $D$. delphis, $L$. albirostris, $B$. borealis, M. bidens, S. scrofa, C. familiaris, F. catus, $M$. furo, M. domesticus, S. harrisii, and O. garnettii) (see "Methods"). Experimental details regarding all of these experiments are described in detail in their respective publications, as well as in the protocols in ArrayExpress.

\section{Pre-processing and read alignment}

Raw sequencing reads (FASTQ files) were aligned to the whole genome of their respective species obtained from Ensembl (v. 81) [64]. The human samples were aligned to the human reference genome used in the 1000 Genomes Project (GRCh37) [65]. Some species were aligned to closely related species' genomes. Specific assemblies and files used for alignment are listed in Additional file 2: Table S4. Finally, the samples of species, for which a full genome was not available (O. garnettii, $H$. glaber, and $M$. furo), were aligned to the reference mitochondrial mtDNA sequence obtained from the NCBI Nucleotide database [66] (Additional file 2: Table S4). All of the raw read files were aligned using BWA (Burrow-Wheeler Aligner) [52] with default parameters. The aligned read files in BAM format were then merged per individual using SAMtools merge [67]. Next, we removed duplicate reads with SAMtools rmdup (keeping only the read with the highest mapping quality score per set of coordinates). Finally, reads aligning to the mtDNA with a mapping quality score of at least 20 were extracted using SAMtools view (parameter $\mathrm{q}=20$ ).

\section{Heteroplasmy detection and data analysis}

The heteroplasmy detection algorithm as well as the analysis performed in this study were implemented in Python 2.7 [68] using the following scientific packages, SciPy [69], Pandas [70], Matplotlib [71], and Pysam (a SAMtools wrapper) and all the code is available in the supplemental data (Additional file 3). Our algorithm processes BAM files, scanning through the entire mitochondrial genome. For each base, it retrieves the set of 
reads covering that base. This read set is first filtered according to two criteria: (1) reads that have a Phred quality score lower than 23 at the position are discarded; and (2) reads for which any of the 5 neighboring base pairs (both directions) has a lower quality than 15 are discarded. Three further criteria are used to call a heteroplasmy on the filtered read set: (1) at least 20 reads should be present in the set; (2) the minor allele (if it exists) should be present on least $15 \%$ of the reads; and (3) the minor allele should be present on at least two reads of each strand.

\section{Heteroplasmy validation}

MtDNA was extracted from $20 \mathrm{mg}$ of flash-frozen liver tissue from each individual of interest, using a protocol adapted from Ahmad et al. [72]. Tissue samples were homogenized in $1 \mathrm{~mL}$ homogenization buffer (100 mM Tris-HCl pH 7.4, $250 \mathrm{mM}$ sucrose, $10 \mathrm{mM}$ EDTA) in a Precellys 24 homogenizer, with conditions $5000^{-3} \times 30^{-30}$ and tubes CK14 (Bertin Technologies). Nuclei and cellular debris were removed by centrifugation (1500 g for $10 \mathrm{~min}$ at $4{ }^{\circ} \mathrm{C}$ ), and the supernatant was centrifuged at $10,000 \mathrm{~g}$ for 10 min at $4{ }^{\circ} \mathrm{C}$ to obtain a crude mitochondrial pellet. Mitochondria were suspended in $480 \mathrm{uL}$ of high salt buffer (Tris $\mathrm{HCl} 10 \mathrm{mM} \mathrm{pH}$ 7.6, $10 \mathrm{mM} \mathrm{KCl,} 10 \mathrm{mM}$ $\mathrm{MgCl} 2,0.4 \mathrm{M} \mathrm{NaCl}$, and $2 \mathrm{mM}$ EDTA) plus $75 \mathrm{uL}$ $10 \%$ SDS, and incubated at $55{ }^{\circ} \mathrm{C}$ for $10 \mathrm{~min}$ for protein denaturation and solubilization. Proteins were precipitated by the addition of $200 \mathrm{uL} 6 \mathrm{M} \mathrm{NaCl}$ and centrifugation at $11,300 \mathrm{~g}$ for $20 \mathrm{~min}$. Finally, the supernatant containing mtDNA was precipitated with two volumes of $100 \%$ ethanol, centrifuged for $10 \mathrm{~min}$ at $10,000 \mathrm{~g}$ at $4{ }^{\circ} \mathrm{C}$, and washed twice with $70 \%$ ethanol. The dried mtDNA pellet was resuspended in $100 \mathrm{uL}$ EB buffer (Qiagen), quantified, and diluted to a final concentration of $100 \mathrm{ng} / \mathrm{uL}$.

PCR primers were designed to amplify two independent mtDNA fragments (400-1000 bp) spanning each heteroplasmic position. A total of $100 \mathrm{ng}$ of mtDNA were used as a template in a $50 \mathrm{uL}$ reaction with Kapa HiFi PCR master mix (Kapa Biosystems) and the following conditions: $95{ }^{\circ} \mathrm{C}$ for $3 \mathrm{~min} ; 20$ cycles of $98{ }^{\circ} \mathrm{C}$ for 20 s, $60{ }^{\circ} \mathrm{C}$ for $30 \mathrm{~s}, 72{ }^{\circ} \mathrm{C}$ for $1 \mathrm{~min}, 72{ }^{\circ} \mathrm{C}$ for $5 \mathrm{~min}, 4{ }^{\circ} \mathrm{C}$ and hold. Sanger sequencing was performed on each amplicon with primers proximal to the heteroplasmy, using at least two different primers per amplicon (Additional file 2: Table S3). Heteroplasmies were considered as robustly validated if both alleles could be clearly detected in the chromatograms of more than $50 \%$ of successful Sanger sequencing reactions (14 positions). For an additional six positions, the minor allele could be detected at lower levels and frequencies, typically in one to three of the reactions (Additional file 2: Table S3).
PCR and sequencing primers for pyrosequencing assays were designed using PyroMark Assay Design 2.0. Each assay was designed to target mitochondrial regions of interest in each species. Primers were designed to ensure high specificity and optimal sequencing length as indicated by their PyroMark quality score. Final PCR reactions included 1.5 units of $\mathrm{MyTaq}^{\mathrm{ma}}$ HS DNA Polymerase (Bioline), $5 \times$ MyTaq Reaction Buffer, 0.27 $\mathrm{uM}$ of each forward and reverse primer, $10 \mathrm{ng}$ of sample DNA and molecular grade water to a final volume of 50 $\mathrm{uL}$ reaction volume. PCR reactions were carried out on an MJ PTC 225 tetrad, 96-well block, in triplicate for each sample including a positive and negative control. PCR cycling conditions were as follows for all assays excluding assay $18 ; 95{ }^{\circ} \mathrm{C}$ for $1 \mathrm{~min}$ initial denaturation; 35 cycles of $95{ }^{\circ} \mathrm{C}$ for $15 \mathrm{~s}$ denaturation, $63{ }^{\circ} \mathrm{C}$ for $15 \mathrm{~s}$ primer annealing, and $72{ }^{\circ} \mathrm{C}$ for $10 \mathrm{~s}$ extension. Assay 18 cycled with an annealing temperature of $50{ }^{\circ} \mathrm{C}$. After a quality check via Agarose Gel Electrophoresis, pyrosequencing was set up and performed on the PSQMA96 machine as per the PyroMark Gold Q96 Reagents Handbook (Qiagen). Following the sequencing runs analysis was performed using PyroMark ID 1.0 via Allele Quantification. Pyrosequencing primer sequences used for each assay can be found in Additional file 2: Table S5.

\section{Coverage and genomic context analysis}

The mean coverage per individual was calculated using SAMtools depth, which provides the coverage for each base pair. The coverage ratio was calculated as the fraction of mtDNA bases that are covered by at least 20 reads. The resulting data are visible in Fig. 2. To assign the genomic context of each heteroplasmic position, the Ensembl Variant Effect Predictor [73] was used. For three species (C. jacchus, M. furo, and O. garnettii), annotations were not available and annotations of closely related species were used (M. mulatta, C. familiaris, and M. mulatta, respectively). The resulting data are visible in Fig. $5 \mathrm{~d}$.

\section{Heteroplasmy visualization}

PRANK [74] with the genomic model was used to generate a multiple alignment of each species' mtDNA. A trimmed species tree extracted from the Ensembl (v. 81) species tree was used as a guide tree. The human mtDNA gene annotation was downloaded from Ensembl (v. 81) BioMart. Jalview [75] was used to visualize heteroplasmic positions for every species displayed on the previously generated alignment (Fig. 3). Jalview was also used to display heteroplasmies in their sequence context (Fig. 4).

\section{Low complexity regions}

There are five low complexity regions in the human mtDNA (66 to 71,303 to 309,514 to $523,12,418$ to 
$12,425$, and 16,184 to 16,193$)$ [18]. To count the number of heteroplasmic positions occurring within these regions across all species, we used the PRANK generated multiple mtDNA alignment to map the non-human heteroplasmic positions to the orthologous human coordinates.

\section{Disease-associated positions}

Human positions were directly compared to MITOMAP annotations to identify potential disease associations. For other species, orthologous human positions for each heteroplasmy were first identified using the UCSC Batch Coordinate Conversion (liftover) tool [76] and then compared to the MITOMAP annotations.

\section{ChIP-seq protein binding assay}

We performed peak detection with the Model-based analysis of ChIP-seq (MACS) tool [77] for a range of experiments covering nine species $(H$. sapiens, $M$. mulatta, $C$. jacchus, $M$. musculus, $R$. norvegicus, $O$. cuniculus, $C$. familiaris, F. catus, and G. gallus) and four different proteins (CEBPA, CTCF, FOXA1, and YY1). No peaks where reported on the mtDNA genome in all cases.

\section{Human contamination test}

To test if the detection method was to subject false positives by cross-species contamination, we simulated human contamination in silico by creating a mixture of $H$. sapiens (human) and R. norvegicus (rat) data. By adding random human sequencing reads to a rat sequence file, which did not contain any heteroplasmies, we created mixture files containing $1 \%$ and $10 \%$ of human DNA. No heteroplasmies were detected by our method in these artificially contaminated files.

\section{Supporting data}

All the data used in this study are accessible on ArrayExpress, under the following accession numbers.

New data from this study:

CEBPA, H3K4me1, H3K27ac, and Histone3 total ChIPseq data: E-MTAB-3933

Previously published data:

HNF4A and CEBPA ChIP-seq data [44]: E-TABM-722 CTCF, SA1, NRSF/REST and H2AK5ac ChIP-seq data [45]: E-MTAB-437

CTCF and YY1 ChIP-seq data [46]: E-MTAB-1511 CEBPA, FOXA1, ONECUT1, and HNF4A ChIP-seq data [47]: E-MTAB-1509

H3K4me3 and H3K27ac ChIP-seq [48]: E-MTAB- 2633

Direct links to raw data and other supporting information are available from http://www.ebi.ac.uk/ research/flicek/publications/FOG17.

\section{Additional files}

Additional file 1: Supplementary figures S1-S3. Figure S1. Detailed coverage data for all individuals. Figure S2. Read coverage of heteroplasmic positions. Figure S3. Coverage data of different ChIP-seq data files. (PDF $20125 \mathrm{~kb}$ )

Additional file 2: Supplementary tables S1-S4. Table S1. Detected heteroplasmies of excluded individuals. Table S2. Detected

heteroplasmies. Table S3. Sanger sequencing and pyrosequencing validation results. Table S4. Additional details for each file. Table S5. Raw pyrosequencing data. (XLS 352 kb)

Additional file 3: Heteroplasmy detection algorithm and analysis in Python. (ZIP $115 \mathrm{~kb}$ )

\section{Acknowledgements}

We thank the Cambridge Institute Genomics and Bioinformatics Core facilities and the Barts and the London Genome Centre for technical assistance. We are grateful to Camille Berthelot, David Thybert, and Graham Ritchie for providing guidance through in-depth discussions, and Romain Studer for providing support with multiple alignments and evolutionary trees. We thank the Duke Lemur Center for providing Otolemur garnettii tissues. This is Duke Lemur Center publication \#1324. Cetacean samples were collected by the UK Cetacean Strandings Investigation Programme, funded by Defra and the Governments of Scotland and Wales.

This research was supported by the European Molecular Biology Laboratory (TR and PF); the EMBL International PhD Programme (TR); the Wellcome Trust (WT095908 [PF] and WT098051 [PF and DTO]); Cancer Research UK (DV, DTO); the European Research Council; and EMBO Young Investigator

Programme (DTO)

\section{Authors' contributions}

PF conceived and supervised the project. TR implemented the code and performed the analysis. DV performed the experiments. $\mathrm{JH}$ provided tissues. TR, DV, DO, and PF contributed to the data analysis and the writing of the manuscript. All authors reviewed and approved this manuscript.

\section{Competing interests}

The authors declare that they have no competing interests.

\section{Ethics approval and consent to participate}

The investigation was approved by the Animal Welfare and Ethics Review Board and followed the Cambridge Institute guidelines for the use of animals in experimental studies under Home Office license PPL 70/7535. For tissue samples collected in external facilities (e.g. commercial suppliers or abattoirs), animals were treated according to local regulations.

\section{Author details}

${ }^{1}$ European Molecular Biology Laboratory, European Bioinformatics Institute, Wellcome Genome Campus, Hinxton, Cambridge CB10 1SD, UK. ${ }^{2}$ Cancer Research UK Cambridge Institute, University of Cambridge, Robinson Way, Cambridge CB2 ORE, UK. ${ }^{3}$ Biological and Biomedical Sciences, North Carolina Central University, Durham, NC 27707, USA. ${ }^{4}$ North Carolina Museum of Natural Sciences, Raleigh, NC 27601, USA. ${ }^{5}$ Wellcome Trust Sanger Institute, Wellcome Genome Campus, Hinxton, Cambridge CB10 1SA, UK.

Received: 9 November 2015 Accepted: 3 June 2016

Published online: 27 June 2016

References

1. Taanman JW. The mitochondrial genome: structure, transcription, translation and replication. Biochim Biophys Acta. 1999;1410:103-23.

2. Kukat C, Wurm CA, Spåhr H, Falkenberg M, Larsson N-G, Jakobs S. Superresolution microscopy reveals that mammalian mitochondrial nucleoids have a uniform size and frequently contain a single copy of mtDNA. Proc Natl Acad Sci U S A. 2011;108:13534-9.

3. Anderson S, Bankier AT, Barrell BG, de Bruijn MH, Coulson AR, Drouin J, et al. Sequence and organization of the human mitochondrial genome. Nature. 1981;290:457-65. 
4. Pakendorf B, Stoneking M. Mitochondrial DNA and human evolution. Annu Rev Genomics Hum Genet. 2005;6:165-83.

5. Stewart JB, Chinnery PF. The dynamics of mitochondrial DNA heteroplasmy: implications for human health and disease. Nat Rev Genet. 2015;16:530-42.

6. Andreu AL, DiMauro S. Current classification of mitochondrial disorders. J Neurol. 2003;250:1403-6.

7. Rossignol R, Faustin B, Rocher C, Malgat M, Mazat J-P, Letellier T. Mitochondrial threshold effects. Biochem J. 2003;370:751-62.

8. Brandon M, Baldi P, Wallace DC. Mitochondrial mutations in cancer. Oncogene. 2006;25:4647-62.

9. Zhidkov I, Livneh EA, Rubin E, Mishmar D. MtDNA mutation pattern in tumors and human evolution are shaped by similar selective constraints. Genome Res. 2009;19:576-80.

10. Coto E, Gómez J, Alonso B, Corao Al, Díaz M, Menéndez M, et al. Late-onset Alzheimer's disease is associated with mitochondrial DNA 7028C/ haplogroup $\mathrm{H}$ and D310 poly-C tract heteroplasmy. Neurogenetics. 2011;12:345-6.

11. Lightowlers RN, Taylor RW, Turnbull DM. Mutations causing mitochondrial disease: What is new and what challenges remain? Science. 2015;349:1494-9.

12. Chinnery PF, Thorburn DR, Samuels DC, White SL, Dahl HHM, Turnbull DM et al. The inheritance of mitochondrial DNA heteroplasmy: Random drift, selection or both? Trends Genet. 2000;16:500-5.

13. Hsieh H, Chiang H, Tsai L, Lai S, Huang N, Linacre A, et al. Cytochrome b gene for species identification of the conservation animals. Forensic Sci Int. 2001;122:7-18.

14. Ketmaier $V$, Bernardini C. Structure of the mitochondrial control region of the Eurasian otter (Lutra lutra; Carnivora, Mustelidae): patterns of genetic heterogeneity and implications for conservation of the species in Italy. J Hered. 2005;96:318-28.

15. Sammler S, Bleidorn C, Tiedemann R. Full mitochondrial genome sequences of two endemic Philippine hornbill species (Aves: Bucerotidae) provide evidence for pervasive mitochondrial DNA recombination. BMC Genomics. 2011;12:35.

16. Sindičić M, Gomerčić T, Galov A, Polanc P, Huber D, Slavica A. Repetitive sequences in Eurasian lynx (Lynx lynx L.) mitochondrial DNA control region. Mitochondrial DNA. 2012;23:201-7.

17. Casane D, Guéride M. Evolution of heteroplasmy at a mitochondrial tandem repeat locus in cultured rabbit cells. Curr Genet. 2002:42:66-72.

18. Hua S, Lu C, Song Y, Li R, Liu X, Quan F, et al. High levels of mitochondrial heteroplasmy modify the development of ovinebovine interspecies nuclear transferred embryos. Reprod Fertil Dev. 2012;24:501-9.

19. Do $M$, Jang WG, Hwang JH, Jang $H$, Kim EJ, Jeong EJ, et al. Inheritance of mitochondrial DNA in serially recloned pigs by somatic cell nuclear transfer (SCNT). Biochem Biophys Res Commun. 2012;424:765-70.

20. Kim KS, Lee SE, Jeong HW, Ha JH. The complete nucleotide sequence of the domestic dog (Canis familiaris) mitochondrial genome. Mol Phylogenet Evol. 1998;10:210-20

21. Machado TS, Macabelli CH, Sangalli JR, Rodrigues TB, Smith LC, Meirelles FV, et al. Real-time PCR quantification of heteroplasmy in a mouse model with mitochondrial DNA of C57BL/6 and NZB/BINJ strains. PLoS One. 2015;10:e0133650.

22. Brown JR, Beckenbach $K$, Beckenbach AT, Smith MJ. Length variation, heteroplasmy and sequence divergence in the mitochondrial DNA of four species of sturgeon (Acipenser). Genetics. 1996;142:525-35.

23. Magnacca KN, Brown MJF. Mitochondrial heteroplasmy and DNA barcoding in Hawaiian Hylaeus (Nesoprosopis) bees (Hymenoptera: Colletidae) BMC Evol Biol. 2010;10:174

24. Klütsch CFC, Seppälä EH, Uhlén M, Lohi H, Savolainen P. Segregation of point mutation heteroplasmy in the control region of dog mtDNA studied systematically in deep generation pedigrees. Int J Legal Med. 2011:125:527-35

25. Greenberg BD, Newbold JE, Sugino A. Intraspecific nucleotide mitochondria DNA sequence variability surrounding the origin of replication in human. Gene. 1983;21:33-49.

26. Irwin JA, Saunier JL, Niederstätter H, Strouss KM, Sturk KA, Diegoli TM, et al. Investigation of heteroplasmy in the human mitochondrial DNA control region: A synthesis of observations from more than 5000 global population samples. J Mol Evol. 2009;68:516-27.

27. White HE, Durston VJ, Seller A, Fratter C, Harvey JF, Cross NCP. Accurate detection and quantitation of heteroplasmic mitochondrial point mutations by pyrosequencing. Genet Test. 2005;9:190-9.
28. Li M, Schönberg A, Schaefer M, Schroeder R, Nasidze I, Stoneking M. Detecting heteroplasmy from high-throughput sequencing of complete human mitochondrial DNA genomes. Am J Hum Genet. 2010;87:237-49.

29. Goto H, Dickins B, Afgan E, Paul IM, Taylor J, Makova KD, et al. Dynamics of mitochondrial heteroplasmy in three families investigated via a repeatable re-sequencing study. Genome Biol. 2011;12:R59.

30. Li M, Stoneking M. A new approach for detecting low-level mutations in next-generation sequence data. Genome Biol. 2012;13:R34.

31. Ding J, Sidore C, Butler TJ, Wing MK, Qian Y, Meirelles O, et al. Assessing mitochondrial DNA variation and copy number in lymphocytes of $\sim 2,000$ Sardinians using tailored sequencing analysis tools. PLoS Genet. 2015;11:e1005306.

32. He Y, Wu J, Dressman DC, lacobuzio-Donahue C, Markowitz SD, Velculescu VE, et al. Heteroplasmic mitochondrial DNA mutations in normal and tumour cells. Nature. 2010:464:610-4.

33. Avital G, Buchshtav M, Zhidkov I, Tuval J, Dadon S, Rubin E, et al. Mitochondrial dna heteroplasmy in diabetes and normal adults: Role of acquired and inherited mutational patterns in twins. Hum Mol Genet. 2012;21:4214-24.

34. Ye K, Lu J, Ma F, Keinan A, Gu Z. Extensive pathogenicity of mitochondrial heteroplasmy in healthy human individuals. Proc Natl Acad Sci. 2014;111:10654-9.

35. Naue J, Hörer S, Sänger T, Strobl C, Hatzer-Grubwieser P, Parson W, et al. Evidence for frequent and tissue-specific sequence heteroplasmy in human mitochondrial DNA. Mitochondrion. 2015:20:82-94.

36. Li M, Schröder R, Ni S, Madea B, Stoneking M. Extensive tissue-related and allele-related mtDNA heteroplasmy suggests positive selection for somatic mutations. Proc Natl Acad Sci. 2015;112:201419651.

37. Diroma MA, Calabrese C, Simone D, Santorsola M, Calabrese FM, Gasparre G, et al. Extraction and annotation of human mitochondrial genomes from 1000 Genomes Whole Exome Sequencing data. BMC Genomics. 2014;15 Suppl 3:S2

38. Hodgkinson A, Idaghdour Y, Gbeha E, Grenier J-C, Hip-ki E, Bruat V, et al. High-resolution genomic analysis of human mitochondrial RNA sequence variation. Science. 2014;344:413-5.

39. Calabrese C, Simone D, Diroma MA, Santorsola M, Gutta C, Gasparre G, et al. MToolBox: A highly automated pipeline for heteroplasmy annotation and prioritization analysis of human mitochondrial variants in high-throughput sequencing. Bioinformatics. 2014;30:3115-7.

40. Taylor RW, Taylor GA, Durham SE, Turnbull DM. The determination of complete human mitochondrial DNA sequences in single cells: implications for the study of somatic mitochondrial DNA point mutations. Nucleic Acids Res. 2001;29:e74.

41. Flensburg C, Kinkel SA, Keniry A, Blewitt M, Oshlack A. A comparison of control samples for ChIP-seq of histone modifications. Front Genet. 2014:5:1-8.

42. Marinov GK, Wang YE, Chan D, Wold BJ. Evidence for site-specific occupancy of the mitochondrial genome by nuclear transcription factors. PLoS One. 2014:9:e84713.

43. Blumberg A, Sri Sailaja B, Kundaje A, Levin L, Dadon S, Shmorak S, et al. Transcription factors bind negatively selected sites within human mtDNA genes. Genome Biol Evol. 2014;6:2634-46.

44. Schmidt D, Wilson MD, Ballester B, Schwalie PC, Brown GD, Marshall A, et al. Five-vertebrate ChIP-seq reveals transcription factor binding. Science. 2010;328:1036-40.

45. Schmidt D, Schwalie PC, Wilson MD, Ballester B, Goncalves $\hat{A}$, Kutter $C$, et al. Waves of retrotransposon expansion remodel genome organization and CTCF binding in multiple mammalian lineages. Cell. 2012;148:335-48.

46. Schwalie PC, Ward MC, Cain CE, Faure AJ, Gilad Y, Odom DT, et al. Co-binding by $Y Y 1$ identifies the transcriptionally active, highly conserved set of CTCF-bound regions in primate genomes. Genome Biol. 2013;14:R148.

47. Ballester B, Medina-Rivera A, Schmidt D, Gonzàlez-Porta M, Carlucci M, Chen $\mathrm{X}$, et al. Multi-species, multi-transcription factor binding highlights conserved control of tissue-specific biological pathways. Elife. 2014;3:1-29.

48. Villar D, Berthelot C, Flicek P, Odom DT, Villar D, Berthelot C, et al. Enhancer evolution across 20 mammalian species article enhancer evolution across 20 mammalian species. Cell. 2015;160:554-66.

49. Schmidt D, Wilson MD, Spyrou C, Brown GD, Odom DT. ChIP-seq: using high-throughput sequencing to discover protein-DNA interactions. Methods. 2009;48:240-8. 
50. Rozowsky J, Euskirchen G, Auerbach RK, Zhang ZD, Gibson T, Bjornson R, et al. PeakSeq enables systematic scoring of ChIP-seq experiments relative to controls. Nat Biotechnol. 2009;27:66-75.

51. Hazkani-Covo E, Zeller RM, Martin W. Molecular poltergeists: mitochondrial DNA copies (numts) in sequenced nuclear genomes. PLoS Genet. 2010;6:e1000834.

52. Li H, Durbin R. Fast and accurate long-read alignment with BurrowsWheeler transform. Bioinformatics. 2010;26:589-95.

53. Lott MT, Leipzig JN, Derbeneva O, Xie HM, Chalkia D, Sarmady M, et al. mtDNA variation and analysis using MITOMAP and MITOMASTER. Curr Protoc Bioinforma. 2013;1:1-26

54. Oh JH, Kim YJ, Moon S, Nam H-Y, Jeon J-P, Lee JH, et al. Genotype instability during long-term subculture of lymphoblastoid cell lines. J Hum Genet. 2013;58:16-20.

55. Tamura K, Nei M. Estimation of the number of nucleotide substitutions in the control region of mitochondrial DNA in humans and chimpanzees. Mol Biol Evol. 1993;10:512-26.

56. Schirmer M, ljaz UZ, D'Amore R, Hall N, Sloan WT, Quince C. Insight into biases and sequencing errors for amplicon sequencing with the Illumina MiSeq platform. Nucleic Acids Res. 2015;43:e37.

57. Tsiatis AC, Norris-Kirby A, Rich RG, Hafez MJ, Gocke CD, Eshleman JR, et al. Comparison of Sanger sequencing, pyrosequencing, and melting curve analysis for the detection of KRAS mutations. J Mol Diagnostics. 2010;12:425-32.

58. Geisler S, Coller J. RNA in unexpected places: long non-coding RNA functions in diverse cellular contexts. Nat Rev Mol Cell Biol. 2013;14:699-712.

59. Lin MF, Kheradpour P, Washietl S, Parker BJ, Pedersen JS, Kellis M. Locating protein-coding sequences under selection for additional, overlapping functions in 29 mammalian genomes. Genome Res. 2011;21:1916-28.

60. Bar-Yaacov D, Avital G, Levin L, Richards AL, Hachen N, Rebolledo Jaramillo $B$, et al. RNA-DNA differences in human mitochondria restore ancestral form of 16S ribosomal RNA. Genome Res. 2013;23:1789-96.

61. Samuels DC, Li C, Li B, Song Z, Torstenson E, Boyd Clay H, et al. Recurrent tissue-specific mtDNA mutations are common in humans. PLoS Genet. 2013:9:e1003929.

62. Kolesnikov N, Hastings E, Keays M, Melnichuk O, Tang YA, Williams E, et al. ArrayExpress update-simplifying data submissions. Nucleic Acids Res. 2015;43:D1113-6.

63. Aldridge S, Watt S, Quail MA, Rayner T, Lukk M, Bimson MF, et al. AHT-ChIPseq: a completely automated robotic protocol for high-throughput chromatin immunoprecipitation. Genome Biol. 2013;14:R124.

64. Cunningham F, Amode MR, Barrell D, Beal K, Billis K, Brent S, et al. Ensembl 2015. Nucleic Acids Res. 2015;43:D662-9.

65. 1000 Genomes Project Consortium. An integrated map of genetic variation from 1,092 human genomes. Nature. 2012;491:56-65.

66. Pruitt KD, Brown GR, Hiatt SM, Thibaud-Nissen F, Astashyn A, Ermolaeva O, et al. RefSeq: An update on mammalian reference sequences. Nucleic Acids Res. 2014;42:756-63.

67. Li H, Handsaker B, Wysoker A, Fennell T, Ruan J, Homer N, et al. The Sequence Alignment/Map format and SAMtools. Bioinformatics. 2009;25:2078-9.

68. van Rossum G. Python tutorial. Tech Rep CS-R9526, Cent voor Wiskd en Inform. 1995.

69. Jones E, Oliphant T, Peterson P. SciPy: Open source scientific tools for Python. 2001.

70. McKinney W. Data structures for statistical computing in Python. In: van der Walt S, Millman J, editors. Proceedings of the 9th Python in Science Conference. Austin, TX: SciPy; 2010. p. 51-6.

71. Hunter JD. Matplotlib: A 2D graphics environment. Comput Sci Eng. 2007:9:99-104.

72. Ahmad S, Ghosh A, Nair DL, Seshadri M. Simultaneous extraction of nuclear and mitochondrial DNA from human blood. Genes Genet Syst. 2007;82:429-32

73. McLaren W, Gil L, Hunt SE, Rait HS, Ritchie GRS, Thormann A, et al. The Ensembl Variant Effect Predictor. Genome Biol. 2016;17:122.

74. Löytynoja A, Goldman N. webPRANK: a phylogeny-aware multiple sequence aligner with interactive alignment browser. BMC Bioinformatics. 2010;11:579.

75. Waterhouse. Jalview Version 2: a multiple sequence alignement editor and analysis workbench. Bioinformatics. 2009;25:1189-91.
76. Hinrichs A, Karolchik D, Baertsch R, Barber G, Bejerano G, Clawson H. The UCSC Genome Browser Database: update 2006. Nucleic Acids Res. 2006;34:D590-8.

77. Zhang Y, Liu T, Meyer CA, Eeckhoute J, Johnson DS, Bernstein BE, et al. Model-based Analysis of ChIP-Seq (MACS). Genome Biol. 2008;9:R137.

\section{Submit your next manuscript to BioMed Central and we will help you at every step:}

- We accept pre-submission inquiries

- Our selector tool helps you to find the most relevant journal

- We provide round the clock customer support

- Convenient online submission

- Thorough peer review

- Inclusion in PubMed and all major indexing services

- Maximum visibility for your research

Submit your manuscript at www.biomedcentral.com/submit
Biomed Central 